\title{
Review Article \\ Vaccines for TB: Lessons from the Past Translating into Future Potentials
}

\author{
Gee Jun Tye, ${ }^{1,2}$ Min Han Lew, ${ }^{1}$ Yee Siew Choong, ${ }^{1,2}$ Theam Soon Lim, ${ }^{1,2}$ \\ Maria Elena Sarmiento, ${ }^{3}$ Armando Acosta, ${ }^{1}$ and Mohd Nor Norazmi ${ }^{1,2,3}$ \\ ${ }^{1}$ Institute for Research in Molecular Medicine, Universiti Sains Malaysia, 11800 Minden, Penang, Malaysia \\ ${ }^{2}$ ADAPT Research Cluster, Centre for Research Initiatives, Clinical \& Health Sciences, Universiti Sains Malaysia, \\ 16150 Kubang Kerian, Kelantan, Malaysia \\ ${ }^{3}$ School of Health Sciences, Universiti Sains Malaysia, Health Campus, 16150 Kubang Kerian, Kelantan, Malaysia \\ Correspondence should be addressed to Gee Jun Tye; geejun@usm.my
}

Received 13 January 2015; Revised 11 May 2015; Accepted 18 May 2015

Academic Editor: Jacek Tabarkiewicz

Copyright (C) 2015 Gee Jun Tye et al. This is an open access article distributed under the Creative Commons Attribution License, which permits unrestricted use, distribution, and reproduction in any medium, provided the original work is properly cited.

\begin{abstract}
Development of vaccines for infectious diseases has come a long way with recent advancements in adjuvant developments and discovery of new antigens that are capable of eliciting strong immunological responses for sterile eradication of disease. Tuberculosis (TB) that kills nearly 2 million of the population every year is also one of the highlights of the recent developments. The availability or not of diagnostic methods for infection has implications for the control of the disease by the health systems but is not related to the immune surveillance, a phenomenon derived from the interaction between the bacteria and their host. Here, we will review the immunology of TB and current vaccine candidates for TB. Current strategies of developing new vaccines against TB will also be reviewed in order to further discuss new insights into immunotherapeutic approaches involving adjuvant and antigens combinations that might be of potential for the control of TB.
\end{abstract}

\section{Introduction}

T-cell vaccination is the administration of antigenic material to produce T-cell immunity against certain diseases. Antigens used in vaccinations range from live viruses or bacteria to peptides of proteins associated with the disease target. Edward Jenner marked the beginning of modern vaccination by using cowpox to give protection against smallpox in humans in 1796. Injecting the harmless form of a disease organism, Jenner utilized the specificity and memory of the acquired immune response to lay the foundation for modern vaccination strategies [1]. For the control of infectious diseases, the core objectives are prophylaxis and therapy. The initial priority of vaccine development has always been prophylaxis, but the development and evaluation of therapeutic vaccines, mainly for chronic infectious diseases and cancer, are gaining momentum. Therapeutic vaccines are substantially more challenging, as diseases such as cancer or chronic infectious diseases would have coexisted with the immune system for a long time and have finally escaped immune surveillance when symptoms are visible. At this stage, almost by definition, tolerance would have been induced $[2,3]$. The exposition to Mycobacterium tuberculosis (M. $t b$ ) produces several outcomes: no infection, latent infection, or active disease $[4,5]$. During the duration of the latency period, $M$. $t b$ goes into a dormant state and would be controlled by the host's immunity. However, this control would slowly bring the immune system to a halt via blockade of regulatory Tcells (Treg) conversion to $\mathrm{T}_{\mathrm{H}} 17$ cells, blockade of $\mathrm{T}_{\mathrm{H}} 2$ cells providing stimulation to memory T-cells $\left(\mathrm{T}_{\mathrm{M}}\right)$, and blockade of costimulatory ligands to avoid activation of effector Tcells $\left(\mathrm{T}_{\mathrm{Eff}}\right)$ [5]. In this context, the immune system loses the capability to contain the reactivation of $M$. $t b$, resulting in overt clinical disease, represented by nearly 8 million of new cases of TB every year. This situation is aggravated by the growing appearance of multidrug resistant TB (MDR$\mathrm{TB}$ ), extensive resistant TB (XDR-TB), and totally resistant TB (TDR-TB). In MDR-TB and XDR-TB, therapy requires alternative, long, expensive, and toxic treatments with low 
success rates while TDR-TB is untreatable. This dire situation now prompts the need for immunotherapies, with special interest in T-cell based vaccines to reactivate cytotoxic T-cells with the capacity to clear disease [6].

\section{An Overview of Mycobacterium tuberculosis and Immunity}

TB is a disease of the respiratory tract that is transmitted through airborne $M$. $t b$. Infection of $M$. $t b$ begins with the deposition in the alveolar spaces of the lungs upon inhaling air with airborne $M$. $t b$. Here, $M$. $t b$ will be phagocytosed by alveolar macrophages (AM) and destroyed with the help of complement activation [7-9]. Cells such as dendritic cells also play a part in this process [10-12] but some of the $M$. $t b$ will be able to escape the process of intracellular destruction by the innate immunity, thus leading to multiplication of the infection and destruction of the AM. Cell debris from the destroyed AM is then ingested by other monocytes which would normally differentiate to other macrophages that would be ready to phagocytose other $M$. $t b$ but does not destroy the internalized $M$. $t b$ from the AM cell debris.

Upon development of acquired immunity (T/B-cell responses), early infected cells would undergo cytotoxicity. Other AM which were secondary to the initial infection will then show inhibited logarithmic bacillary growth where the infection now goes into a dormant state [13]. During the dormant/latent state, $M$. $t b$ escapes immune surveillance via several strategies. It is capable of inhibiting both phagosome maturation and autophagy as well as translocating from the phagolysosomes to the cytosol of the AM [14, 15]. $M$. $t b$ is also capable of downregulating proinflammatory cytokines, gamma-interferon (IFN-gamma), and gammainterferon receptor (IFN-gammaR) which is crucial T/B-cell responses [16]. Production of anti-inflammatory cytokines such as IL-10, IL-1Ra, and IL-4 and transforming growth factor beta (TGF- $\beta$ ) on the other hand antagonizes the protective $\mathrm{T}_{\mathrm{H}} 1$ responses by IFN-gamma and IL-1 $\beta$ as well as inducing regulatory T-cells (Tregs) [16-20].

The development of immune-surveillance escape mechanisms does not stop at the cytokine level. Processing pathways of major histocompatibility complex (MHC) class I, MHC class II, and CD1 molecule which presents antigen have also been observed to be inhibited by $M$. $t b$. The mechanism with which MHC class I molecule is inhibited is yet to be clearly understood but data shows the possibility of pathogen associated molecular patterns [21], especially the $19 \mathrm{kDa}$ lipoprotein antigen known as $\mathrm{LpqH}$ from $M$. $t b$ to inhibit phagosome maturation and thus limit the availability of antigenic peptides from $M$. $t b$ to be presented on MHC class I molecules [22-24]. MHC class II molecules which are important for stimulating $\mathrm{CD}^{+}$T-cells in order to generate $T_{M}$ against diseases were more clearly observed to be attenuated by the LpqH from $M$. $t b$. It acts as a PAMP and triggers activation of macrophages through toll like receptor (TLR) 2. However, this chronic exposure to LpqH and M. $t b$ infection causes inhibition of IFN-gamma-induced regulation genes $(46 \%)$, which play important roles in presentation of antigen on MHC class II molecules [25, 26]. This eventually leads to downregulation of antigen presentation and reduced activation of $\mathrm{CD} 4{ }^{+}$T-cells $[27,28]$.

With the combination of these mechanisms, $M . t b$ would normally succeed in evading the host immune system and lays dormant or slowly multiplies within the necrotic granuloma of the infected lungs (consisting of dysregulated granuloma turnover, liquefactive necrosis, and pathological scarring). The lesion formations in the granuloma are heterologously infected by $M$. tuberculosis, giving rise to the production of the $\mathrm{CD}^{+}$and $\mathrm{CD}^{+}$cells. The microenvironment of the human lesions determines the different responses caused by the $M$. $t b$ cells depending on the location of the bacteria in the outer lesion wall [29]. The condition within these granulomas not only provides an excellent breeding ground for multiplication of $M$. $t b$ but also serves as a spreading ground due to access to the airways of the lungs [30]. Therefore, the immunity conferred at the early stages of the infection would only serve as a vague warning sign to the patient, and in most cases many $\mathrm{TB}$ patients go undiagnosed until reaching active state where the immune system is already unable to retaliate against the disease. This problem is aggravated by the fact that there is no gold standard diagnostics available for latent TB: that is, about $10 \%$ of these numbers go into active TB state [5]. Although new interferon gamma release assays (IGRA) which detect M. $t b$ specific antigen release of IFN-gamma are capable of producing more accurate results than the Mantoux test, there are still problems with false positive test results [31]. Therefore, current treatment of TB has poor prognosis as treatment is delayed due to the lack in diagnostics and the inability to stimulate an immunological response when TB enters the active state. This fact also strengthens the need to develop improved vaccines which would generate stronger and lasting immunological responses against $\mathrm{TB}$ be it for prophylaxis or therapy.

Current trends of understanding TB have also progressed as far as the involvement of other nonconventional T-cells (iNKT, CD1 restricted T-cells, $\gamma \delta$ T-cells, Th17 cells, etc. [32]), presentation of peptides by HLA-E molecules [33, 34], and recognition of glycolipids/glycoproteins by CD1 restricted Tcells [35]. These have all been extensively reviewed in the cited publications and go to show that immunity generated as a result of $M$. $t b$ infection not only confers the CD4, CD8, and B-cells immunity but also is extended towards other parts of the immunity due to its capacity to avoid immune surveillance.

\section{Current Prophylactic TB Vaccines and Their Developments}

Historically, TB vaccine has been targeted towards prophylaxis/protective immunization with Bacille de Calmette et Guérin (BCG) which was first recognized in 1931 [36, 37]. BCG, a vaccine made of live attenuated Mycobacterium bovis (mycobacterium strain that causes $\mathrm{TB}$ in cattle), is used worldwide but is now shown to be unable to protect adults with pulmonary $\mathrm{TB}$ and adolescents despite showing 
beneficial protection in children [38]. Increasing numbers of active TB is also fueled by HIV coinfection [39-43]. HIV targets the $\mathrm{CD} 4^{+}$T-cells which plays an important role in protection against $\mathrm{TB}$ as discussed in the previous chapter. Due to this coinfection, HIV compromises T-cell based immunity generated against TB, leading to accelerated reactivation of latent TB [44].

In order to tackle such problems, new candidate vaccines are emerging from around the globe. These candidate vaccines are intended to provide not only stronger immunological responses against $M$. tb but also long lasting responses which will require stimulation of memory $\mathrm{T}$ - and B-cell responses [38].

One of the many examples of current emerging TB vaccines is the MVA85A vaccine, which is based on Ag85A antigen expressed by modified Vaccinia Ankara virus as a subunit vaccine. Ag85A is a mycolyltransferase found in $M$. $t b$ during its dormant stage to mediate the transesterification of diacylglycerol as acyl donors to form lipid storage bodies causing persistence of infection [45]. The MVA vaccine, which was developed as a smallpox vaccine in 1983, was first used in several animal models, which produced immunogenic responses and provides protection against BCG vaccinated animals. Safety studies conducted on BCG treated patients also showed positive results in a phase I clinical trial [46]. The vaccination protocol as of then was $B C G$ vaccination followed by MVA $85 A$ vaccination and then fowl pox expressing Ag85A (FP85A) vaccination in a primeboost strategy. This strategy showed a marked increase in Ag85A-specific $\mathrm{CD}^{+}$T-cells after vaccination with FP85A [47]. Although phase I studies were successful, further clinical studies in a phase IIb randomized trial showed no efficacy compared to placebo [48]. The trial recruited an extensive number of patients (2797 infants, 1399 allocated to MVA85A and 1398 allocated to placebo) in Cape Town, South Africa, but the authors only found that the vaccine was safe and generated modest immunological responses. However, efficacy was absent and further investigations are currently ongoing in order to dissect the findings.

Although the MVAg85A phase IIb failed to show improved efficacy, efforts in developing vaccines against TB should be increased and clinical trials must be designed in a manner to incorporate current understanding such as nonconventional T-cells and presentation of glycolipids/glycoproteins to CD1 restricted T-cells. This is due to the fact that, in phase IIb trial of MVAg85A, protective efficacy was shown by BCG vaccination and thus increased efficacy was not seen in such a short period. The lack of a nonBCG vaccinated group also points towards the need for better clinical trial designs [38].

Other prophylactic vaccines, which are currently in the pipeline, are listed in Table 1. One of the many interesting vaccines includes a recombinant BCG (rBCG) designed to express a lysin from Listeria monocytogenes, which would promote presentation of antigens delivered to the host called VPM1002 [49]. This vaccine was cleverly designed to increase the capacity of BCG vaccine to promote $\mathrm{CD} 8^{+} \mathrm{T}$-cell responses. Listeriolysin acts within the infected macrophages to induce apoptosis as well as translocation of antigens into the cytoplasm, thus increasing available antigens to be presented by MHC class I molecule. VPM1002 has since concluded its phase I trial showing safety, immunogenicity, and stimulation of both B- and T-cell responses against antigens from the vaccine [50]. However, studies as such would require further investigation and investigators would need to learn from results obtained from the MV85A clinical trials.

We have so far discussed examples of non- $M$. $t b$ vaccines which were used in vaccination against $\mathrm{TB}$. The notion of immunizing with attenuated live $M$. $t b$ was also used by several groups to design new vaccines against TB. Despite worries that the attenuated $M . t b$ is a viable microorganism that has intrinsic potential risk to produce active disease, but this type of vaccine is of great potential. For obvious reasons, the use of a live attenuated $M$. $t b$ would provide all the necessary antigens and the mycobacterium itself would act as an adjuvant as the presence of numerous types of liposaccharides is capable of triggering the innate immunity, thus increasing generation of proinflammatory cytokines such as TNF- $\alpha$, IL-2, and IFN-gamma.

One such example is the $\mathrm{SO} 2$ vaccine, a mutant strain of $M$. tb MT103 that has a disrupted phoP gene $[51,52]$. The phoP gene was shown to play an important role in the virulence of M. $t b$ strain MT103 and this disruption reduces the potential of replication significantly [53]. The $\mathrm{SO} 2$ vaccine presents several key antigens from $M$. $t b$ which have been shown to be crucial in conferring protection compared to BCG. The ESAT-6 antigen, for example, was shown to be highly immunodominant in a $M$. $t b$ challenge study conducted in guinea pigs which was not found in BCG vaccination [54-56]. Therefore, the use of live attenuated $M . t b$ as vaccine delivers a potential alternative.

Another notable vaccine is the fusion protein HyVac4 (H4), which consists of the mycobacterial antigens Ag85B and TB10.4, which is administered with the adjuvant IC31 or DDA/MPL in BCG-induced individual. H4 was shown in a preclinical study with 6- to 8-week-old female F1 crossing of inbred male C57BL/6 and female Balb/c mice to have effectively boosted and prolonged immunity induced by BCG with immune response dominated by IFN $\gamma / \mathrm{TNF} \alpha / \mathrm{IL}-2$ or TNF $\alpha /$ IL2 producing CD4 T-cells [57]. Phase II clinical trials have been announced by Aeras in March 2014 and would enroll 990 adolescents.

$\mathrm{H} 56$ which is a fusion protein vaccine consisting of Ag85B and ESAT-6 is very similar to $\mathrm{H} 4$ as it also works in order to boost the immunological responses generated by BCG. In a preclinical study with BCG inoculated cynomolgus macaques, boosting with H56/IC31 resulted in efficient containment of $M$. tuberculosis infection and reduced rates of clinical disease. This was measured by clinical parameters, inflammatory markers, and improved survival of the animals compared with BCG alone [58]. H56 is developed by Statens Serum Institut (SSI) and has since collaborated with AERAS for a clinical trial. Other notable vaccines which have shown to be of great potential have been incorporated in Table 1 above. 
TABLE 1: Candidate vaccine with emerging protective responses and its brief description.

\begin{tabular}{ll}
\hline TB vaccine & Vaccine details \\
\hline MVA85A [45] & $\begin{array}{l}\text { Modified Vaccinia Ankara virus } \\
\text { expressing Ag85A }\end{array}$ \\
\hline VPM1002 [50] & rBCG expressing listeriolysin \\
\hline AdAg85A [89] & Adenovirus expressing Ag85A \\
\hline IIKEPLUS [90] & $\begin{array}{l}\text { Mycobacterium smegmatis mutant } \\
\text { that expresses } \text { M. tb esx-3 genes } \\
\text { which is capable of inducing central } \\
\text { memory responses }\end{array}$ \\
\hline SO2 [52] & $\begin{array}{l}\text { Mutant } M . \text { tb strain that has phoP } \\
\text { deleted }\end{array}$ \\
\hline Hybrid 1+ IC31 [91] & $\begin{array}{l}\text { Fusion of ESAT-6 and Ag85B in } \\
\text { adjuvant IC31 }\end{array}$ \\
\hline H4 [57] & $\begin{array}{l}\text { Ag85B and TB10.4 administered } \\
\text { with IC31 or DDA/MPL }\end{array}$ \\
\hline H56 [58] & $\begin{array}{l}\text { Ag85B and ESAT-6 administered } \\
\text { with IC31 }\end{array}$ \\
\hline rBCG30 [92, 93] & $\begin{array}{l}\text { 30-kDa major secretory protein of } \\
\text { Mycobacterium tuberculosis }\end{array}$ \\
\hline M72 + AS01/AS02 [94] & $\begin{array}{l}\text { Fusion protein of Rv1196 and } \\
\text { Rv0125 in AS01 or AS02 adjuvant }\end{array}$ \\
\hline
\end{tabular}

\section{Current Therapeutic TB Vaccines and Their Developments}

Prophylactic vaccines for TB, as previously discussed, showed that only BCG was capable of providing protection but is incomplete. Due to the fact that the protection conferred is incomplete, reactivation of $\mathrm{TB}$ in latently infected patients poses a great risk and eradication of disease still proves challenging [59]. With the discovery of MDR, XDR, and TDR strains of $M$. $t b$, treatment of active TB remains as one of the top priorities, which will complement prophylactic vaccinations in order to eradicate TB. Without drugs capable of combating these $M$. $t b$ strains, nearly 2 million of the world population are killed yearly with some patients not even receiving any treatment [60]. New avenues and combination of drugs in clinical trials showed some form of positive results like meropenem-clavulanate in combination with linezolidcontaining regimens to treat XDR TB [61]. However, a new line of thought in using therapeutic vaccines has emerged with the introduction of several different candidates.

Currently there are only a handful of therapeutic vaccines that are in the development pipeline. An example of these vaccines is an inactivated whole-cell Mycobacterium vaccae ( $M$. vaccae). This vaccine was first introduced in 1985 to boost BCG vaccinations instead of being used in treating leprosy $[62,63]$. Inactivated $M$. vaccae possesses similar immunodominant antigens to $M$. $t b$, thus showing an improved generation of antigen-specific lymphocytes responses especially in HIV-patients with TB. Despite showing efficacy inducing $\mathrm{T}_{\mathrm{H}} 1$ responses, antibody responses, especially IgG against mycobacterial antigens, were also shown to escalate in these vaccinated
TABLE 2: Candidate emerging therapeutic TB vaccines and its brief description.

\begin{tabular}{ll}
\hline Vaccine & Vaccine details \\
\hline RUTI [68] & $\begin{array}{l}\text { A liposome that encapsulates } \\
\text { detoxified } M \text {. tb fragments }\end{array}$ \\
\hline $\begin{array}{l}\text { Mycobacterium } \\
\text { indicus pranii (MIP) } \\
{[95]}\end{array}$ & $\begin{array}{l}\text { A live saprophytic mycobacterium } \\
\text { administered via aerosol route }\end{array}$ \\
\hline CSU-F36 [67] & $\begin{array}{l}\text { A fusion of Rv1411 (TLR-2 agonist) and } \\
\text { ESAT-6 protein }\end{array}$ \\
\hline
\end{tabular}

patients [64]. When one dose of inactivated $10^{9} \mathrm{M}$. vaccae was administered in combination with chemotherapy treatment, significant clinical improvements were seen in nonHIV patients in a clinical trial conducted in Uganda [65]. Another trial with three doses of $M$. vaccae also showed clinical improvements in non-HIV, chemotherapy treated patients [66]. This progress however meant that treatment with chemotherapy is still crucial.

Other potential therapeutic vaccines are also in the pipeline but one similarity between the vaccines is that they augment responses generated by chemotherapy or are used to control latent infection postchemotherapy (Table 2). Therapeutic vaccines such as CSU-F36 which is a fusion of a TLR-2 agonist and ESAT- 6 antigen act as a standalone vaccine which have the potential of generating cytotoxic T-cell lymphocytes responses as strong level IL-12 was generated [67]. However, this vaccination has only managed to withstand a mild dose of aerosol $M$. $t b$ challenge.

RUTI is a new form of immunotherapy involving the use of detoxified and liposome Mycobacterium tuberculosis cell fragments in the vaccine regimen (Table 2). Preclinical studies have shown that RUTI treatment has the tendency to improve the chemotherapy with increased efficiency against chronic disorders caused by the Mycobacterium tuberculosis mouse model (C57BL/6 and DBA/2 strains) and guinea pigs [68]. This one-month vaccination strategy utilises the bactericidal effect provided by the chemotherapy to synergistically kill the active bacilli growth and suppress the inflammatory responses generated locally. The inoculation of RUTI can be implemented after the chemotherapy in order to prevent the reactivation of the latent bacilli. The intranasal administration of the RUTI vaccine in mice contributes to the reduction in the bacillus cell counts and balanced Th1/Th2/Th3 responses without toxicity. RUTI is advantageous over other $\mathrm{TB}$ vaccines due to the protective properties given by the specific CD8 T-cells and humoral responses induced during the treatment. This enables the immune system to fight against a broad range of antigen with antigenspecific antibody productions upon RUTI vaccination [69].

RUTI extract has successfully elicited the pronounced immune responses caused by the recombinant mycobacterial antigens [70]. Significant protection was demonstrated in the mice sera treated with RUTI regimen in SCID mice [71]. The RUTI vaccine has shown prophylactic effect as therapeutic vaccine against tuberculosis. In C57BL mouse model, the viable bacilli count was significantly reduced in both lung 
and spleens after 4 weeks upon vaccinations. Stronger protection was observed for lung as compared to spleen cells after 9-month vaccination. Besides, the guinea pig survived longer by giving 5 -week vaccination prior to challenge. RUTI vaccination can potentially be a prophylactic treatment to reduce the risk of tuberculosis infections [72]. During phase II clinical trial, a randomised, placebo-controlled study has shown a reasonably safe vaccine which is tolerable and is immunogenic in human subjects with latent tuberculosis $[69,72]$.

The development of an effective $\mathrm{TB}$ vaccine has been challenging on the path of the licensure of the therapeutic product with proven safety and effectiveness. To design a potent prophylactic vaccine, animal models have been used extensively during vaccine development. However, successful clinical trials are required to investigate the immune response generated during an actual condition of the TB infection. Further studies are required to understand immune responses that occur naturally upon infection. To circumvent this issue, the tracking of the global TB vaccine development is important and this can be achieved by encouraging Stop TB partnership to gather vaccine pipelines to acknowledge the current candidate vaccine profile and novel therapeutic strategies. The combination of priming, booster, and therapeutic vaccines may provide protection before and after TB antigen exposure during TB infection, which can be further enhanced by rapid diagnostic approach and adjunct drug treatment against multidrug resistant TB [73, 74].

\section{Future Directions into TB Vaccines}

Current vaccinations in the pipeline are much skewed towards protective vaccination and therapy in combination with chemotherapy. This current trend is very plausible and in the light of the results obtained, be it positive or negative, it would only point towards the possible control of TB. However, we are still far away from sterile eradication of TB. In order to improve on discoveries which have already been made, we would like to underline several key factors that we think would assist in development of vaccines with enhanced efficacy.

One of the key factors that we and others think would be of crucial importance towards generating a better vaccine is the role of B-cells and antibodies [5]. Like all vaccines against infectious disease, that is, hepatitis B. virus, antibodies play the dominant role in providing initial protection against incoming infection. Antibodies are binders that not only are specific towards their targets but also recruit many arms of the immune system (NK-cells, neutrophils, and so forth) to the site of infection, thus increasing the chance of sterile eradication. Although we have discussed the ability of $M$. $t b$ to evade this mode of eradication, we think that by introducing a vaccine capable of triggering Th2 responses and an appropriate antigen especially during the latent stage might generate antibodies which are targeted against pulmonary $M$. $t b$. The discovery of downregulated invariant natural killer T-cells (iNKT cells) in peripheral blood of TB patients [75] also points towards the possibility of using antibodies as a mode of targeting latent infection and activation of iNKT cells via $\alpha$-galactosylceramide ( $\alpha$-GalCer) [32, 76] would be able to lead to the destruction of latently infected cells. However, as mentioned by Nunes-Alves et al., the use of $\alpha$-GalCer has yet to be explored. This however can be substituted by other iNKT cells activator such as the minor lipid species that copurifies with $\beta$-GlcCer in mammals [77]. Aside from inducing antibody responses, the use of appropriate antigen is key to generating cytotoxic antigen-specific $\mathrm{CD} 4^{+}$and $\mathrm{CD} 8^{+} \mathrm{T}$-cells. As we have observed in current vaccines being developed, they were mostly targeted against Ag85A, Ag85B, and ESAT-6. These targets are all very immunodominant and if so, with latent TB constantly stimulating similar Tcell clones and results showing upregulation of Tregs in patients, there will also be Tregs that are specifically targeted towards suppressing T-cell responses against these antigens. This situation is often noticed in chronic diseases as well as in cancer whereby antigen-specific Tregs are often found to have superior suppressive capacity, thus leading to suppression of T-cell responses [78]. We speculate that this might be one of the reasons why results obtained from clinical trials are inferior compared to results obtained from animal studies. Generating T-cell responses against $M$. $t b$ which is yet to be suppressed would require the search of new antigens which are constitutively expressed yet less immunodominant. One example of such possible antigen is Mtb32 that, despite being less studied, has shown to be a promising antigen for generating $\mathrm{CD}^{+}$and $\mathrm{CD}^{+}$T-cell responses in pre- and postexposure in $M$. $t b$ mouse models $[79,80]$.

Another key factor in designing a potential vaccine would be the use of an adjuvant which would provide depot effects, stimulate the innate immunity to provide a proinflammatory environment, and skew Th responses [81]. A combination of a Squalene/Tween-80 emulsion, 2 TLR agonist, an MHC class I target peptide, an MHC class II helper peptide, and IFN-gamma has recently showed generation of high levels of antigen-specific $\mathrm{CD}^{+}{ }^{+} \mathrm{T}$-cells which not only are cytotoxic but also were shown to generate memory T-cells. Responses generated by this combination (CASAC adjuvant) not only were against foreign antigens (ovalbumin) but also were capable of mounting responses towards selfantigen (tyrosinase-related protein 2). Responses generated were capable of eradicating a B16 mouse melanoma challenge. The responses generated could also be recalled after a 50-day resting period [82].

One final key factor that we should factor in is to use antibodies that would deplete or block suppressive signals such as programmed-death 1 (PD-1), cytotoxic T-lymphocyte antigen 4 (CTLA-4), and ultimately Tregs. PD-1, CTLA-4, and Tregs are all correlated in suppressing T-cell responses. In a recent study to develop cancer vaccines, $\mathrm{PD}-1$ and CTLA4 have shown to be present on tumour infiltrating lymphocytes (TIL), antigen-specific $\mathrm{CD} 8^{+} \mathrm{T}$-cells. This presence was regulated by Tregs which at the end causes dysfunction of cytotoxic T-cells that were infiltrating tumours. In a comparative study, dual blockade of PD-1 and CTLA-4 restored the dysfunction of these TIL and caused $100 \%$ rejection of tumour [83]. This could be a very interesting avenue to pursue in order to possibly restore functions of reactive T-cells that 
might have been generated in the initial infection stage of TB as described previously.

Based on these lines of thoughts, we suggest the possibility of developing new vaccines based on the use of an adjuvant such as CASAC in combination with new antigens which is of less immunodominance and pretreatment with antibody that depletes or blocks suppressive responses such as anti-PD1 and anti-CTLA- 4 .

On a similar train of thoughts, we think that using heat shock proteins (HSP) as an immunogenic carrier could be of potential benefit [84-86]. HSP are a group of proteins that is recognized by the human immune system and expressed during inflammation. The human immune system has a natural autoimmunity towards HSP, particularly HSP60 here, whereby $\mathrm{CD} 4^{+} \mathrm{T}$-cells responses would be generated. HSP 60 showed positive preclinical results in multiple occasions as an adjuvant which will provide help towards both $\mathrm{CD} 8^{+} \mathrm{T}$ cells and B-cells due to activation of the $\mathrm{CD} 4^{+} \mathrm{T}$ helper cell in diseases such as murine CMV [85], Meningitides [87], and West Nile virus [88]. Therefore, with the right selection of $M$. $t b$ antigen or attenuated whole $M$. $t b$ cell in combination with potent adjuvant and the right pretreatment, we think that generation of a new line of vaccine for TB would not be a far cry away in the hope of eliminating TB.

\section{Conflict of Interests}

There is no conflicting interest among all authors.

\section{Acknowledgment}

The authors would like to acknowledge the contribution of the Universiti Sains Malaysia for funding the work via Research Universiti Individual Grant Scheme (RUI: 1001/ CIPPM/812119).

\section{References}

[1] P. J. Delves and I. M. Roitt, Roitt's Essential Immunology, WileyBlackwell, Chichester, UK, 2011.

[2] R. D. Schreiber, L. J. Old, and M. J. Smyth, "Cancer immunoediting: integrating immunity's roles in cancer suppression and promotion," Science, vol. 331, no. 6024, pp. 1565-1570, 2011.

[3] M. Dougan and G. Dranoff, "Immune therapy for cancer," Annual Review of Immunology, vol. 27, pp. 83-117, 2009.

[4] K. Dheda, S. K. Schwander, B. Zhu, R. N. Van Zyl-Smit, and Y. Zhang, "The immunology of tuberculosis: from bench to bedside," Respirology, vol. 15, no. 3, pp. 433-450, 2010.

[5] T. H. M. Ottenhoff and S. H. E. Kaufmann, "Vaccines against tuberculosis: where are we and where do we need to go?" PLoS Pathogens, vol. 8, no. 5, Article ID e1002607, 2012.

[6] S. C. Gilbert, "T-cell-inducing vaccines-what's the future," Immunology, vol. 135, no. 1, pp. 19-26, 2012.

[7] L. S. Schlesinger, C. G. Bellinger-Kawahara, N. R. Payne, and M. A. Horwitz, "Phagocytosis of Mycobacterium tuberculosis is mediated by human monocyte complement receptors and complement component C3," Journal of Immunology, vol. 144, no. 7, pp. 2771-2780, 1990.

[8] H. Diaz-Silvestre, P. Espinosa-Cueto, A. Sanchez-Gonzalez et al., "The $19-\mathrm{kDa}$ antigen of Mycobacterium tuberculosis is a major adhesin that binds the mannose receptor of THP-1 monocytic cells and promotes phagocytosis of mycobacteria," Microbial Pathogenesis, vol. 39, no. 3, pp. 97-107, 2005.

[9] C. S. Hirsch, J. J. Ellner, D. G. Russell, and E. A. Rich, "Complement receptor-mediated uptake and tumor necrosis factoralpha-mediated growth inhibition of Mycobacterium tuberculosis by human alveolar macrophages," Journal of Immunology, vol. 152, no. 2, pp. 743-753, 1994.

[10] N. N. Driessen, R. Ummels, J. J. Maaskant et al., "Role of phosphatidylinositol mannosides in the interaction between mycobacteria and DC-SIGN," Infection and Immunity, vol. 77, no. 10, pp. 4538-4547, 2009.

[11] T. Geijtenbeek, S. J. van Vliet, E. A. Koppel et al., "Mycobacteria target DC-SIGN to suppress dendritic cell function," Journal of Experimental Medicine, vol. 197, no. 1, pp. 7-17, 2003.

[12] L. Tailleux, N. Pham-Thi, A. Bergeron-Lafaurie et al., "DCSIGN induction in alveolar macrophages defines privileged target host cells for mycobacteria in patients with tuberculosis," PLoS Medicine, vol. 2, no. 12, article e381, 2005.

[13] R. van Crevel, T. H. M. Ottenhoff, and J. W. M. van der Meer, "Innate immunity to Mycobacterium tuberculosis," Clinical Microbiology Reviews, vol. 15, no. 2, pp. 294-309, 2002.

[14] M. G. Gutierrez, S. S. Master, S. B. Singh, G. A. Taylor, M. I. Colombo, and V. Deretic, "Autophagy is a defense mechanism inhibiting BCG and Mycobacterium tuberculosis survival in infected macrophages," Cell, vol. 119, no. 6, pp. 753-766, 2004.

[15] N. van der Wel, D. Hava, D. Houben et al., "M. tuberculosis and $M$. leprae translocate from the phagolysosome to the cytosol in myeloid cells," Cell, vol. 129, no. 7, pp. 1287-1298, 2007.

[16] E. Sahiratmadja, B. Alisjahbana, T. De Boer et al., "Dynamic changes in pro- and anti-inflammatory cytokine profiles and gamma interferon receptor signaling integrity correlate with tuberculosis disease activity and response to curative treatment," Infection and Immunity, vol. 75, no. 2, pp. 820-829, 2007.

[17] K. E. Dahl, H. Shiratsuchi, B. D. Hamilton, J. J. Ellner, and Z. Toossi, "Selective induction of transforming growth factor $\beta$ in human monocytes by lipoarabinomannan of Mycobacterium tuberculosis," Infection and Immunity, vol. 64, no. 2, pp. 399-405, 1996.

[18] V. A. Boussiotis, E. Y. Tsai, E. J. Yunis et al., "IL-10-producing $\mathrm{T}$ cells suppress immune responses in anergic tuberculosis patients," The Journal of Clinical Investigation, vol. 105, no. 9, pp. 1317-1325, 2000.

[19] Z. Toossi, P. Gogate, H. Shiratsuchi, T. Young, and J. J. Ellner, "Enhanced production of TGF-beta by blood monocytes from patients with active tuberculosis and presence of TGF-beta in tuberculous granulomatous lung lesions," The Journal of Immunology, vol. 154, no. 1, pp. 465-473, 1995.

[20] R. J. North, "Mice incapable of making IL-4 or IL-10 display normal resistance to infection with Mycobacterium tuberculosis," Clinical and Experimental Immunology, vol. 113, no. 1, pp. 55-58, 1998.

[21] E. Pampe, "Contagious tuberculosis in village schools and its effect on BCG vaccinated and unvaccinated children," Zeitschrift für Tuberkulose, vol. 106, no. 6, pp. 334-338, 1955.

[22] A. A. R. Tobian, N. S. Potter, L. Ramachandra et al., "Alternate class I MHC antigen processing is inhibited by Tolllike receptor signaling pathogen-associated molecular patterns: Mycobacterium tuberculosis 19-kDa lipoprotein, CpG DNA, and lipopolysaccharide," Journal of Immunology, vol. 171, no. 3, pp. 1413-1422, 2003. 
[23] D. P. Simmons, D. H. Canaday, Y. Liu et al., "Mycobacterium tuberculosis and TLR2 agonists inhibit induction of type I IFN and class I MHC antigen cross processing by TLR9," The Journal of Immunology, vol. 185, no. 4, pp. 2405-2415, 2010.

[24] A. Baena and S. A. Porcelli, "Evasion and subversion of antigen presentation by Mycobacterium tuberculosis: review article," Tissue Antigens, vol. 74, no. 3, pp. 189-204, 2009.

[25] R. K. Pai, M. E. Pennini, A. A. R. Tobian, D. H. Canaday, W. H. Boom, and C. V. Harding, "Prolonged toll-like receptor signaling by Mycobacterium tuberculosis and its 19-kilodalton lipoprotein inhibits gamma interferon-induced regulation of selected genes in macrophages," Infection and Immunity, vol. 72, no. 11, pp. 6603-6614, 2004.

[26] R. K. Pai, M. Convery, T. A. Hamilton, W. Henry Boom, and C. V. Harding, "Inhibition of IFN- $\gamma$-induced class II transactivator expression by a $19-\mathrm{kDa}$ lipoprotein from Mycobacterium tuberculosis: a potential mechanism for immune evasion," The Journal of Immunology, vol. 171, no. 1, pp. 175-184, 2003.

[27] Z. Hmama, R. Gabathuler, W. A. Jefferies, G. De Jong, and N. E. Reiner, "Attenuation of HLA-DR expression by mononuclear phagocytes infected with Mycobacterium tuberculosis is related to intracellular sequestration of immature class II heterodimers," Journal of Immunology, vol. 161, no. 9, pp. 48824893, 1998.

[28] E. H. Noss, R. K. Pai, T. J. Sellati et al., “Toll-like receptor 2dependent inhibition of macrophage class II MHC expression and antigen processing by $19-\mathrm{kDa}$ lipoprotein of Mycobacterium tuberculosis," Journal of Immunology, vol. 167, no. 2, pp. 910-918, 2001.

[29] C. E. Barry, H. I. Boshoff, V. Dartois et al., "The spectrum of latent tuberculosis: rethinking the biology and intervention strategies," Nature Reviews Microbiology, vol. 7, no. 12, pp. 845$855,2009$.

[30] K. Dheda, H. Booth, J. F. Huggett, M. A. Johnson, A. Zumla, and G. A. W. Rook, "Lung remodeling in pulmonary tuberculosis," Journal of Infectious Diseases, vol. 192, no. 7, pp. 1201-1210, 2005.

[31] M. Pai, J. Minion, H. Sohn, A. Zwerling, and M. D. Perkins, "Novel and improved technologies for tuberculosis diagnosis: progress and challenges," Clinics in Chest Medicine, vol. 30, no. 4, pp. 701-716, 2009.

[32] C. Nunes-Alves, M. G. Booty, S. M. Carpenter, P. Jayaraman, A. C. Rothchild, and S. M. Behar, "In search of a new paradigm for protective immunity to TB," Nature Reviews Microbiology, vol. 12, no. 4, pp. 289-299, 2014.

[33] K. E. van Meijgaarden, M. C. Haks, N. Caccamo et al., "Human $\mathrm{CD}^{+} \mathrm{T}$-cells recognizing peptides from Mycobacterium tuberculosis (Mtb) presented by HLA-E have an unorthodox Th2like, multifunctional, Mtb inhibitory phenotype and represent a novel human T-cell subset," PLOS Pathogens, vol. 11, no. 3, Article ID e1004671, 2015.

[34] S. A. Joosten, K. E. van Meijgaarden, P. C. van Weeren et al., "Mycobacterium tuberculosis peptides presented by HLA-E molecules are targets for human CD8+ T-cells with cytotoxic as well as regulatory activity," PLoS Pathogens, vol. 6, no. 2, Article ID e1000782, 2010.

[35] I. van Rhijn, A. Kasmar, A. de Jong et al., "A conserved human $\mathrm{T}$ cell population targets mycobacterial antigens presented by CD1b," Nature Immunology, vol. 14, no. 7, pp. 706-713, 2013.

[36] A. Calmette, "Preventive vaccination against tuberculosis with BCG," Proceedings of the Royal Society of Medicine, vol. 24, no. 11, pp. 1481-1490, 1931.
[37] W. G. Pereira, "PATIENTS BCG vaccination, tuberculosis and immunization Calmette home," Hospital (Rio de Janeiro, Brazil), vol. 36, no. 5, pp. 681-698, 1949.

[38] I. M. Orme, "Vaccine development for tuberculosis: current progress," Drugs, vol. 73, no. 10, pp. 1015-1024, 2013.

[39] S. Charalambous, J. H. Day, K. Fielding, K. M. de Cock, G. J. Churchyard, and E. L. Corbett, "HIV infection and chronic chest disease as risk factors for bacterial pneumonia: a casecontrol study," AIDS, vol. 17, no. 10, pp. 1531-1537, 2003.

[40] G. J. Churchyard, K. Fielding, S. Charalambous et al., "Efficacy of secondary isoniazid preventive therapy among HIV-infected Southern Africans: time to change policy?" AIDS, vol. 17, no. 14, pp. 2063-2070, 2003.

[41] E. L. Corbett, "HIV and tuberculosis: surveillance revisited," International Journal of Tuberculosis and Lung Disease, vol. 7, no. 8, p. 709, 2003.

[42] E. L. Corbett, S. Charalambous, K. Fielding et al., "Stable incidence rates of tuberculosis (TB) among human immunodeficiency virus (HIV)-negative South African gold miners during a decade of epidemic HIV-associated TB," Journal of Infectious Diseases, vol. 188, no. 8, pp. 1156-1163, 2003.

[43] E. L. Corbett, C. J. Watt, N. Walker et al., "The growing burden of tuberculosis: global trends and interactions with the HIV epidemic," Archives of Internal Medicine, vol. 163, no. 9, pp. 1009-1021, 2003.

[44] P. Andersen and T. M. Doherty, "The success and failure of BCG-implications for a novel tuberculosis vaccine," Nature Reviews Microbiology, vol. 3, no. 8, pp. 656-662, 2005.

[45] A. A. Elamin, M. Stehr, R. Spallek, M. Rohde, and M. Singh, "The Mycobacterium tuberculosis Ag85A is a novel diacylglycerol acyltransferase involved in lipid body formation," Molecular Microbiology, vol. 81, no. 6, pp. 1577-1592, 2011.

[46] H. McShane, A. A. Pathan, C. R. Sander et al., "Recombinant modified vaccinia virus Ankara expressing antigen 85A boosts BCG-primed and naturally acquired antimycobacterial immunity in humans," Nature Medicine, vol. 10, no. 11, pp. 1240-1244, 2004.

[47] H. McShane, A. A. Pathan, C. R. Sander, N. P. Goonetilleke, H. A. Fletcher, and A. V. S. Hill, "Boosting BCG with MVA85A: the first candidate subunit vaccine for tuberculosis in clinical trials," Tuberculosis, vol. 85, no. 1-2, pp. 47-52, 2005.

[48] M. D. Tameris, M. Hatherill, B. S. Landry et al., "Safety and efficacy of MVA85A, a new tuberculosis vaccine, in infants previously vaccinated with BCG: a randomised, placebo-controlled phase 2b trial," The Lancet, vol. 381, no. 9871, pp. 1021-1028, 2013.

[49] L. Grode, P. Seiler, S. Baumann et al., "Increased vaccine efficacy against tuberculosis of recombinant Mycobacterium bovis bacille Calmette-Guérin mutants that secrete listeriolysin," Journal of Clinical Investigation, vol. 115, no. 9, pp. 2472-2479, 2005.

[50] L. Grode, C. A. Ganoza, C. Brohm, J. Weiner III, B. Eisele, and S. H. E. Kaufmann, "Safety and immunogenicity of the recombinant BCG vaccine VPM1002 in a phase 1 open-label randomized clinical trial," Vaccine, vol. 31, no. 9, pp. 1340-1348, 2013.

[51] C. Martin, A. Williams, R. Hernandez-Pando et al., "The live Mycobacterium tuberculosis phoP mutant strain is more attenuated than BCG and confers protective immunity against tuberculosis in mice and guinea pigs," Vaccine, vol. 24, no. 17, pp. 3408-3419, 2006.

[52] D. Aguilar, E. Infante, C. Martin, E. Gormley, B. Gicquel, and R. Hernandez Pando, "Immunological responses and protective 
immunity against tuberculosis conferred by vaccination of Balb/C mice with the attenuated Mycobacterium tuberculosis (phoP) SO2 strain," Clinical \& Experimental Immunology, vol. 147, no. 2, pp. 330-338, 2007.

[53] J. G. Asensio, C. Maia, N. L. Ferrer et al., "The virulenceassociated two-component PhoP-PhoR system controls the biosynthesis of polyketide-derived lipids in Mycobacterium tuberculosis," The Journal of Biological Chemistry, vol. 281, no. 3, pp. 1313-1316, 2006.

[54] A. S. Pym, P. Brodin, L. Majlessi et al., "Recombinant BCG exporting ESAT-6 confers enhanced protection against tuberculosis," Nature Medicine, vol. 9, no. 5, pp. 533-539, 2003.

[55] A. S. Pym, P. Brodin, R. Brosch, M. Huerre, and S. T. Cole, "Loss of RD1 contributed to the attenuation of the live tuberculosis vaccines Mycobacterium bovis BCG and Mycobacterium microti," Molecular Microbiology, vol. 46, no. 3, pp. 709-717, 2002.

[56] Y. Lu, Y. Xu, E. Yang, C. Wang, H. Wang, and H. Shen, "Novel recombinant BCG coexpressing Ag85B, ESAT-6 and Rv2608 elicits significantly enhanced cellular immune and antibody responses in C57BL/6 mice," Scandinavian Journal of Immunology, vol. 76, no. 3, pp. 271-277, 2012.

[57] R. Billeskov, T. T. Elvang, P. L. Andersen, and J. Dietrich, “The HyVac4 subunit vaccine efficiently boosts BCG-primed antimycobacterial protective immunity," PLoS ONE, vol. 7, no. 6, Article ID e39909, 2012.

[58] P. L. Lin, J. Dietrich, E. Tan et al., "The multistage vaccine H56 boosts the effects of BCG to protect cynomolgus macaques against active tuberculosis and reactivation of latent Mycobacterium tuberculosis infection," Journal of Clinical Investigation, vol. 122, no. 1, pp. 303-314, 2012.

[59] S. H. E. Kaufmann, "Future vaccination strategies against tuberculosis: thinking outside the Box," Immunity, vol. 33, no. 4, pp. 567-577, 2010.

[60] I. Monedero and J. A. Caminero, "MDR-/XDR-TB management: what it was, current standards and what is ahead," Expert Review of Respiratory Medicine, vol. 3, no. 2, pp. 133-145, 2009.

[61] S. de Lorenzo, J. W. Alffenaar, G. Sotgiu et al., "Efficacy and safety of meropenem-clavulanate added to linezolid-containing regimens in the treatment of MDR-/XDR-TB," European Respiratory Journal, vol. 41, no. 6, pp. 1386-1392, 2013.

[62] C. A. Brown, I. N. Brown, and S. Swinburne, "The effect of oral Mycobacterium vaccae on subsequent responses of mice to BCG sensitization," Tubercle, vol. 66, no. 4, pp. 251-260, 1985.

[63] R. G. Navalkar, S. D. Chaparas, C. K. Lakshiminarayana, and M. V. Kanchana, "Antigenic evaluation of Mycobacterium vaccae in relation to Mycobacterium leprae," International Journal of Leprosy, vol. 48, no. 4, pp. 388-396, 1980.

[64] G. M. Bahr, M. A. Shaaban, M. Gabriel et al., "Improved immunotherapy for pulmonary tuberculosis with Mycobacterium vaccae," Tubercle, vol. 71, no. 4, pp. 259-266, 1990.

[65] J. L. Johnson, R. M. Kamya, A. Okwera et al., "Randomized controlled trial of Mycobacterium vaccae immunotherapy in non-human immunodeficiency virus-infected Ugandan adults with newly diagnosed pulmonary tuberculosis," Journal of Infectious Diseases, vol. 181, no. 4, pp. 1304-1312, 2000.

[66] D. Dlugovitzky, G. Fiorenza, M. Farroni, C. Bogue, C. Stanford, and J. Stanford, "Immunological consequences of three doses of heat-killed Mycobacterium vaccae in the immunotherapy of tuberculosis," Respiratory Medicine, vol. 100, no. 6, pp. 10791087, 2006.
[67] B. Wang, M. Henao-Tamayo, M. Harton et al., "A toll-like receptor-2-directed fusion protein vaccine against tuberculosis," Clinical and Vaccine Immunology, vol. 14, no. 7, pp. 902-906, 2007.

[68] P. J. Cardona and I. Amat, "Origin and development of RUTI, a new therapeutic vaccine against Mycobacterium tuberculosis infection," Archivos de Bronconeumologia, vol. 42, no. 1, pp. 2532, 2006.

[69] P.-J. Cardona, "RUTI: a new chance to shorten the treatment of latent tuberculosis infection," Tuberculosis, vol. 86, no. 3-4, pp. 273-289, 2006.

[70] O. Gil, C. Vilaplana, E. Guirado et al., "Enhanced gamma interferon responses of mouse spleen cells following immunotherapy for tuberculosis relapse," Clinical and Vaccine Immunology, vol. 15, no. 11, pp. 1742-1744, 2008.

[71] E. Guirado, O. Gil, N. Cáceres, M. Singh, C. Vilaplana, and P. J. Cardona, "Induction of a specific strong polyantigenic cellular immune response after short-term chemotherapy controls bacillary reactivation in murine and guinea pig experimental models of tuberculosis," Clinical and Vaccine Immunology, vol. 15, no. 8, pp. 1229-1237, 2008.

[72] C. Vilaplana, E. Montané, S. Pinto et al., "Double-blind, randomized, placebo-controlled Phase I Clinical Trial of the therapeutical antituberculous vaccine RUTI," Vaccine, vol. 28, no. 4, pp. 1106-1116, 2010.

[73] M. I. Gröschel, S. A. Prabowo, P.-J. Cardona, J. L. Stanford, and T. S. van der Werf, "Therapeutic vaccines for tuberculosis-a systematic review," Vaccine, vol. 32, no. 26, pp. 3162-3168, 2014.

[74] J. Weiner and S. H. E. Kaufmann, "Recent advances towards tuberculosis control: vaccines and biomarkers," Journal of Internal Medicine, vol. 275, no. 5, pp. 467-480, 2014.

[75] C. J. Montoya, J. C. Cataño, Z. Ramirez, M. T. Rugeles, S. B. Wilson, and A. L. Landay, "Invariant NKT cells from HIV1 or Mycobacterium tuberculosis-infected patients express an activated phenotype," Clinical Immunology, vol. 127, no. 1, pp. 1-6, 2008.

[76] A. Chackerian, J. Alt, V. Perera, and S. M. Behar, "Activation of NKT cells protects mice from tuberculosis," Infection and Immunity, vol. 70, no. 11, pp. 6302-6309, 2002.

[77] P. J. Brennan, R. V. Tatituri, C. Heiss et al., "Activation of iNKT cells by a distinct constituent of the endogenous glucosylceramide fraction," Proceedings of the National Academy of Sciences, vol. 111, no. 37, pp. 13433-13438, 2014.

[78] A. Schmidt, N. Oberle, and P. H. Krammer, "Molecular mechanisms of treg-mediated T cell suppression," Frontiers in Immunology, vol. 3, article 51, 2012.

[79] S. S. Ahn, B. Y. Jeon, S. J. Park et al., "Nonlytic Fc-fused IL7 synergizes with Mtb32 DNA vaccine to enhance antigenspecific T cell responses in a therapeutic model of tuberculosis," Vaccine, vol. 31, no. 27, pp. 2884-2890, 2013.

[80] S.-S. Ahn, B.-Y. Jeon, K.-S. Kim et al., "Mtb32 is a promising tuberculosis antigen for DNA vaccination in pre- and postexposure mouse models," Gene Therapy, vol. 19, no. 5, pp. 570$575,2012$.

[81] M. J. Bevan, "Understand memory, design better vaccines," Nature Immunology, vol. 12, no. 6, pp. 463-465, 2011.

[82] J. W. Wells, C. J. Cowled, F. Farzaneh, and A. Noble, "Combined triggering of dendritic cell receptors results in synergistic activation and potent cytotoxic immunity," Journal of Immunology, vol. 181, no. 5, pp. 3422-3431, 2008. 
[83] J. Duraiswamy, K. M. Kaluza, G. J. Freeman, and G. Coukos, "Dual blockade of PD-1 and CTLA-4 combined with tumor vaccine effectively restores T-cell rejection function in tumors," Cancer Research, vol. 73, no. 12, pp. 3591-3603, 2013.

[84] S. Könen-Waisman, A. Cohen, M. Fridkin, and I. R. Cohen, "Self heat-shock protein (hsp60) peptide serves in a conjugate vaccine against a lethal pneumococcal infection," Journal of Infectious Diseases, vol. 179, no. 2, pp. 403-413, 1999.

[85] O. Rouvio, T. Dvorkin, H. Amir-Kroll et al., "Self HSP60 peptide serves as an immunogenic carrier for a CTL epitope against persistence of murine cytomegalovirus in the salivary gland," Vaccine, vol. 23, no. 27, pp. 3508-3518, 2005.

[86] F. J. Quintana, P. Carmi, F. Mor, and I. R. Cohen, "DNA fragments of the human $60-\mathrm{kDa}$ heat shock protein (HSP60) vaccinate against adjuvant arthritis: identification of a regulatory HSP60 peptide," Journal of Immunology, vol. 171, no. 7, pp. 3533-3541, 2003.

[87] H. Amir-Kroll, L. Riveron, M. E. Sarmiento, G. Sierra, A. Acosta, and I. R. Cohen, "A conjugate vaccine composed of a heat shock protein 60 T-cell epitope peptide (p458) and Neisseria meningitidis type B capsular polysaccharide," Vaccine, vol. 24, no. 42-43, pp. 6555-6563, 2006.

[88] O. Gershoni-Yahalom, S. Landes, S. Kleiman-Shoval et al., "Chimeric vaccine composed of viral peptide and mammalian heat-shock protein 60 peptide protects against West Nile virus challenge," Immunology, vol. 130, no. 4, pp. 527-535, 2010.

[89] H. Zhou, X. Chen, Y. Ji et al., "Construction of recombinant adenovirus expressing Ag85B of Mycobacterium bovis and its cellular immunoproperties in mice," Wei Sheng Wu Xue Bao, vol. 50, no. 6, pp. 811-816, 2010.

[90] K. A. Sweeney, D. N. Dao, M. F. Goldberg et al., "A recombinant Mycobacterium smegmatis induces potent bactericidal immunity against Mycobacterium tuberculosis," Nature Medicine, vol. 17, no. 10, pp. 1261-1268, 2011.

[91] E. M. Agger, I. Rosenkrands, A. W. Olsen et al., "Protective immunity to tuberculosis with Ag85B-ESAT-6 in a synthetic cationic adjuvant system IC31," Vaccine, vol. 24, no. 26, pp. 5452-5460, 2006.

[92] T. P. Gillis, M. V. Tullius, and M. A. Horwitz, "rBCG30-induced immunity and cross-protection against Mycobacterium leprae challenge are enhanced by boosting with the Mycobacterium tuberculosis 30-kilodalton antigen 85B," Infection and Immunity, vol. 82, no. 9, pp. 3900-3909, 2014.

[93] M. A. Horwitz and G. Harth, "A new vaccine against tuberculosis affords greater survival after challenge than the current vaccine in the guinea pig model of pulmonary tuberculosis," Infection and Immunity, vol. 71, no. 4, pp. 1672-1679, 2003.

[94] C. L. Day, M. Tameris, N. Mansoor et al., "Induction and regulation of T-cell immunity by the novel tuberculosis vaccine M72/AS01 in South African adults," American Journal of Respiratory and Critical Care Medicine, vol. 188, no. 4, pp. 492-502, 2013.

[95] A. Gupta, F. J. Ahmad, U. D. Gupta, M. Natarajan, V. M. Katoch, and S. Bhaskar, "Protective efficacy of Mycobacterium indicus pranii against tuberculosis and underlying local lung immune responses in guinea pig model," Vaccine, vol. 30, no. 43, pp. 6198-6209, 2012. 


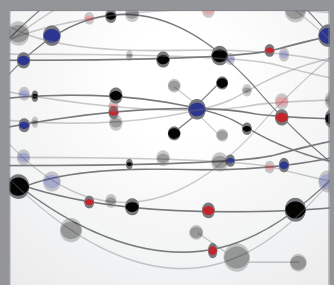

The Scientific World Journal
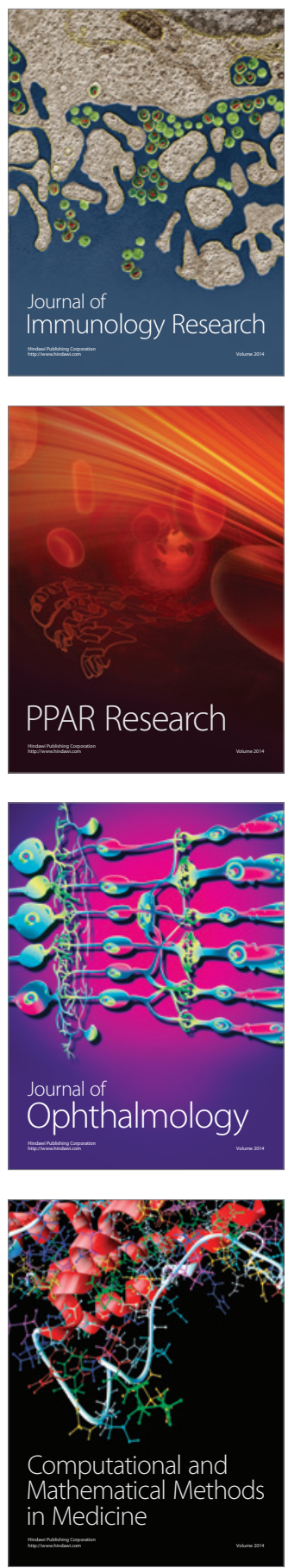

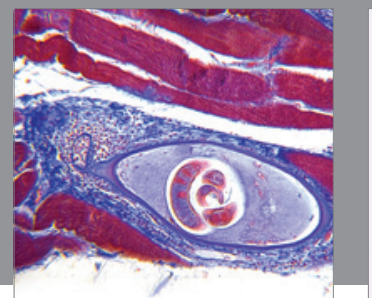

Gastroenterology

Research and Practice
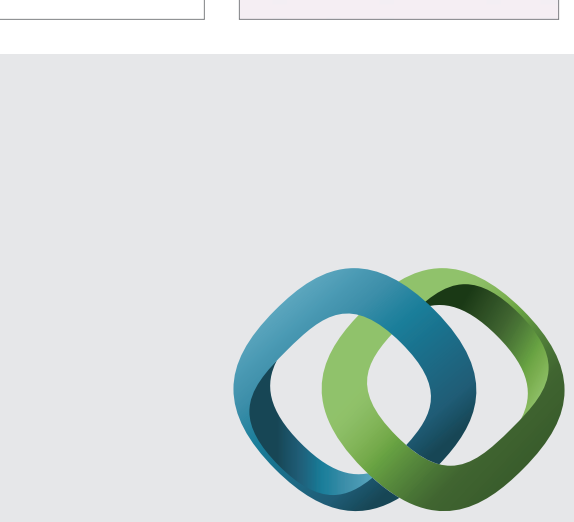

\section{Hindawi}

Submit your manuscripts at

http://www.hindawi.com
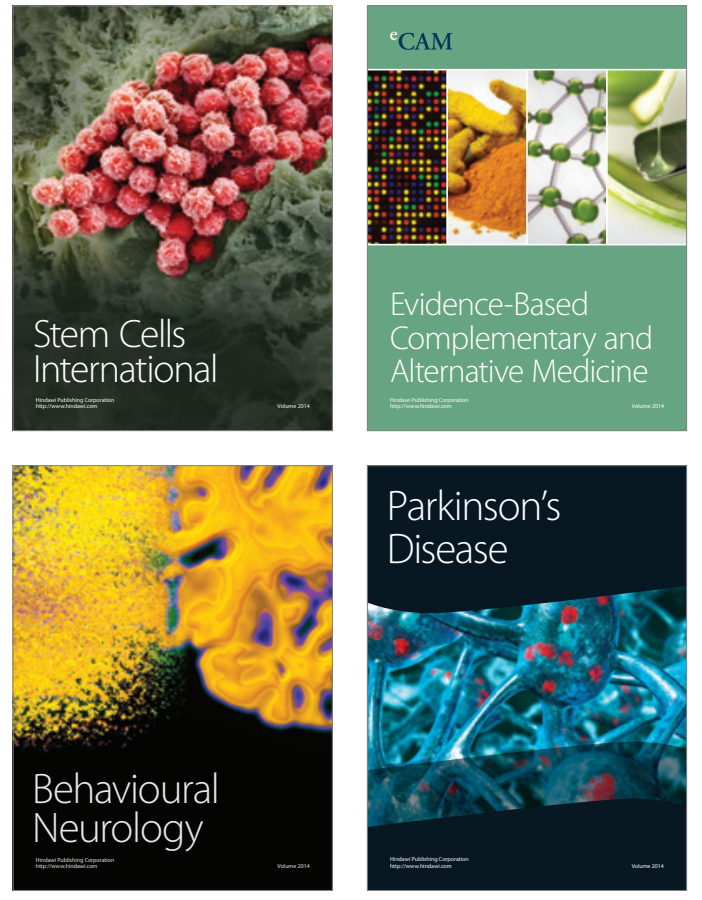
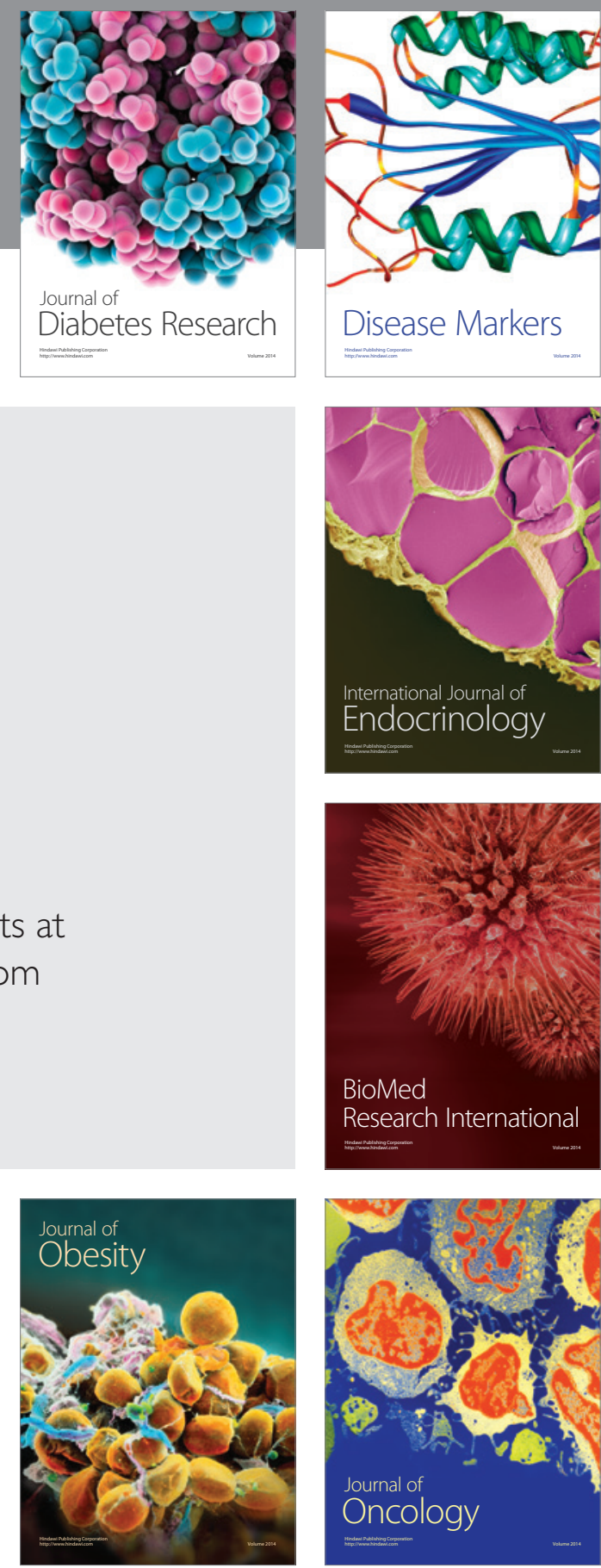

Disease Markers
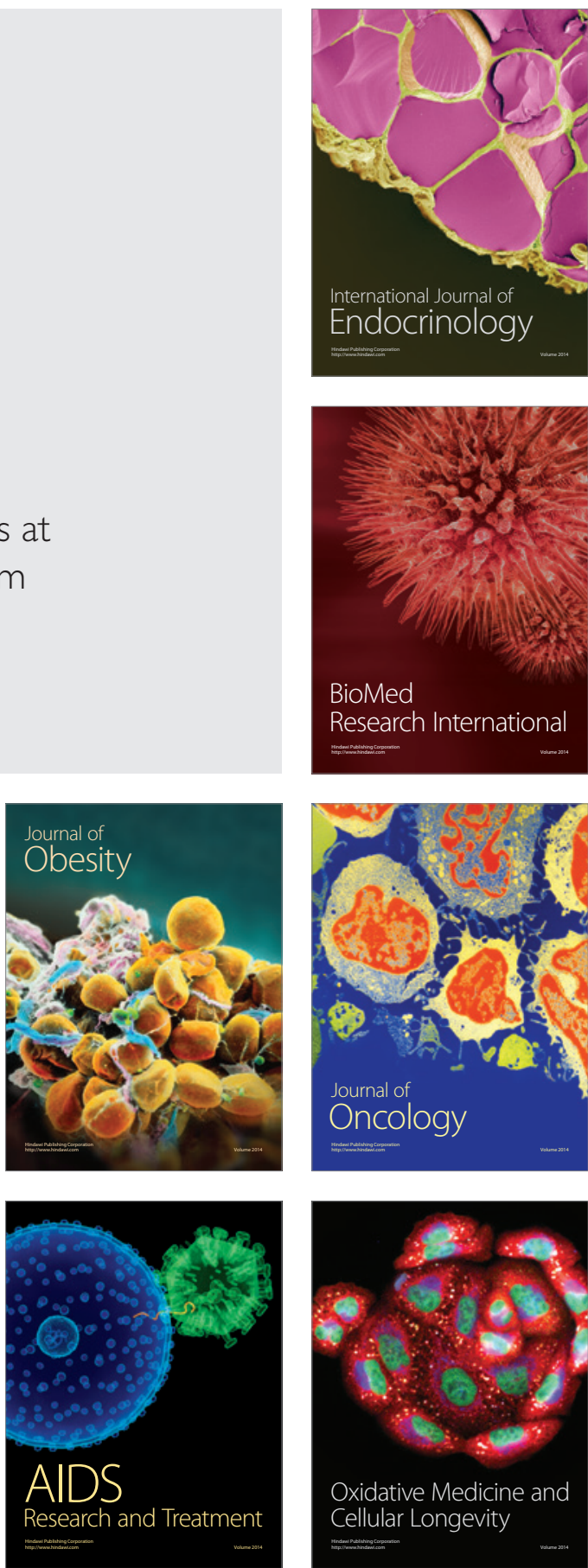